\title{
Machine Intelligence Techniques in VLSI Hardware
}

\author{
Ashish Chauhan, Laxmi Singh, Sanjeev Kumar Gupta
}

\begin{abstract}
A brief guide to storage primarily dependent strategies to simulate machine intelligence in VLSI equipment, explaining the difficulties accompanied by the advantages. The application of programming methods in VLSI hardware can be both difficult and reasonable. Deep architectures, stratified temporal reminiscences and memory networks square measure a number of up-to-date approaches in this analysis area. This methodology was built to follow the low-level intelligence challenges and seeks to provide climbable approaches to highlevel intelligence problems such as thin writing and debate.
\end{abstract}

Keywords-component; formatting; style; styling; insert (keywords)

\section{INTRODUCTION}

The neocortex is manifest in the brain of human, accounting for seventy-six percent of the brain volume, and could be seen as a dwelling unit involved in the mechanism of intelligence functionalities such as "sensory perception, technological revolution of motor commands, spatial reasoning, conscious thinking and language". The discovery that cerebral mantle structures by protocols and software integration have always been the basis for the greater part of state-of-the-art computer engineering programs and theory [1]. Intelligent Function Region Units usually required to be conducted in situations with high levels of natural variability, making specific brain features exceptionally progressed. On the other hand, the wide variety of neurons in the cerebral mantle and their interdependencies with each other end up making the brain operationally very diversified.

As a consequence, the replication of cognitive cape is extremely complex and challenging. The human brain is a very normal and educated in practicality and structure, each cell in a human brain has developed into a system and is equipped to perform a very particular task. The communication among the components begins in the versatility of the human brain to understand and react to specialized cognitive activity tasks. The activity of the system represents a hierarchical structure wherever the level of cognitive systems and decision-making mechanisms is the highest part of the pyramid.[2].

The human brain is extremely unique and innovative in form and function. Each cell in the human brain has progressed in architecture and is equipped to handle quite a

Revised Manuscript Received on 14 August, 2019.

Dr. AshishChauhan, Department of Electronics \& Communication Engineering, Rabindranath Tagore University,Bhopal, Madhya Pradesh, India.

Dr. Laxmi Singh, Department of Electronics \& Communication Engineering, Rabindranath Tagore University, Bhopal, Madhya Pradesh, India.

Dr. Sanjeev Kumar Gupta, Department of Electronics \& Communication Engineering, Rabindranath Tagore University,Bhopal, Madhya Pradesh, India. large mission. Contact among the modules lies in the power of the human brain to recognize and respond to specific cognitive activities. The relation of the unit follows a different graduated relationship, where tactile processes form the basic part of the sequence of commands and decision-making techniques to the uppermost portion of the command structure. Nonetheless, even with the difficulty of unpredictable nature, since the mid-1950s, researchers have wanted to simulate the neuron and cortex by means of "computational, human, algorithmic, biomimetic, neuromorphic circuits" as well. Computational, cognitive, or human brain memory capability has inspired researchers to replicate brain capacity and skill in the artificial intelligence process. Artificial intelligence systems are discovered in software or hardware and are often based on computational or logical argumentation of mental capacities, such as artificial neural network, fuzzy logic, evolutionary computing, Bayesian networks, expert systems, case-based reasoning, and behavior-based artificial intelligence". We seek interest in knowledge-based problems, model awareness issues, enhancement issues and flexible management issues. Artificial intelligence chips for computers have an extensive range of interests for data, but existing machinery use is constrained to a small functional scalable design and an uncertain amount of sources of information. For reference, the development of an artificial neural network involves multiple neural hubs and carries requiring various amplifiers and storage components. The wide area is required to position such nodes, with an increase in the number of neural hubs, and the number of neural node interdependencies in different layers appears to be small. It adds to the difficulty of cell networking in a small chip zone, thus large area specifications for speakers and storage devices restrict the volume of the device. In contrast, fuzzy logic chip utilization with a large number of sources of information is impracticable due to device unpredictable nature.

\section{MACHINE INTELLIGENCE TECHNIQUES}

Even with reliability difficult lties, since the early 1950s, scientists have decided to base the vegetative cell and hence the cortex across computational, biochemical, repetitive, biomimetic and xeromorphic processes, techniques, mental and memory capacities of human brains, which have inspired investigators to imitate brain function and capacity in artificial intelligent systems [3][4][5][6].

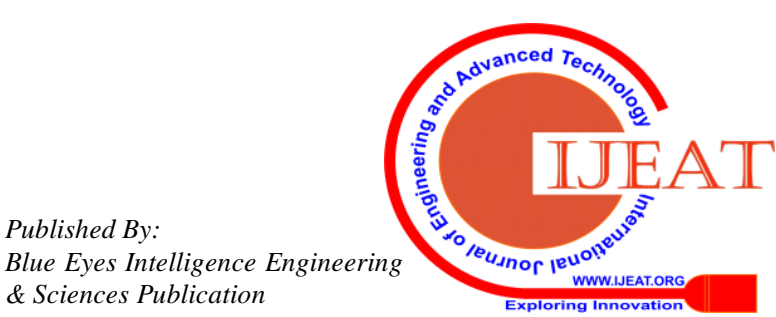


Intelligent superseding models square measure implemented in software or hardware and often extracted by computational and theoretical brain capacity simulation, square measurement examples of artificial neural networks, numerical reasoning, biological process computation, Bayesian networks, intelligent structures, case-based justification, or actions dependent mostly on computing. Applications of data-focused mostly on problems, issues related to pattern recognition, difficulties related to change and responsive. Competent artificial smart hardware chips have a number of advantages over computer device implementations; furthermore, the existing hardware design square measurements are restricted to poor purposeful performance and a minimal set of inputs. As a correlated networks involves many neural nodes with weights that require multiple amplifiers and storage modules.Massive space is needed to place these components, and with an increase in the spectrum of neural entities, the amount of neural node linkages in a number of layers is large. It adds to the difficulty of cell routing in a very small chip area, while the huge space demands of the amplifiers and storage modules narrow the measurements of the system, numerical logic chip deployment is conducted in the same direction with a significant volume of inputs is likewise that, hardware performance credits.

Fig 1:The gradable existence of the "gradable temporal storage networks" in human cognition and thought cannot occur without knowing the meaning of memory. Human cognition has relentlessly attracted the attention of neuroscientists over the last seventy years and has only recently attacked the imagination of artificial intelligence investigators. In the so-called intelligent machines that we all recognize these days, the beginning of memory is essential to the theory of smart computation, without modeling some activity or simulating some training process, which is perhaps difficult.

Because it is so obvious that knowledge is important, the idea of memory being the foundation of wisdom is a lower percentage in terms of understanding and study. Some upto-date system awareness is trying to emulate information victimization knowledge in a methodology or the reverse.Rather than just pursuing an associated algorithmic strategy to diagnosis intelligence, it is evident by looking at the descriptive nature of the human brain and the neuron network that human reminiscences and psychological features of the area unit, coordinated in a gradable sort of way, will be modeled and imitated in order to develop new strategies for diagnosis intelligence. Perhaps one of the key solutions to network evaluation is that the supposed artificial neural networks pursuing problem-solving patternrecognition and optimization via coaching network weights across a controlled and semi-supervised methodology.

Period Hubel-Wiesel designs (MHWA) can be a deep structure composed of overlapping layers of function indicators and native pooling of choices. A variety of solutions for the "MHWA region module, convolution networks, HMAX and its versions". Nonetheless, the sensible design of neural replacement networks in equipment is not a quick and easy process involved in a number of cases and is not a feasible job. degree illustration, the development of artificial neural

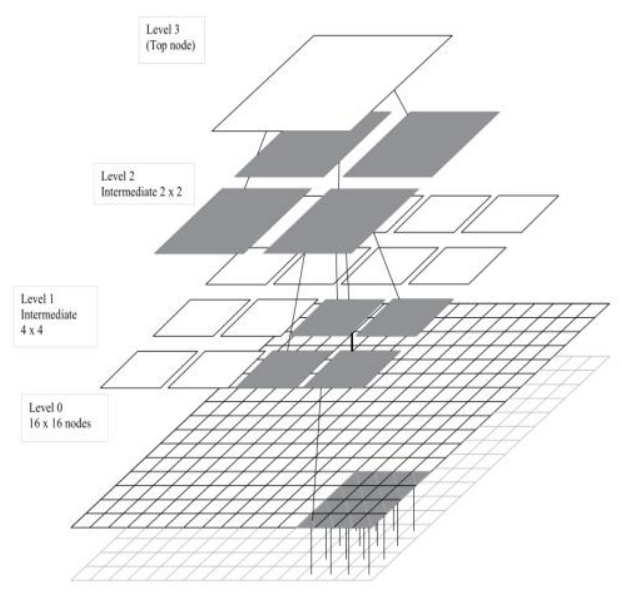

\section{Fig.1. the hierarchical nature of the hierarchical temporal memory networks}

\section{PROPOSED TECHNIQUE\& RESULTS}

With an improvement in the number of artificial cells (or nodes) the volume of cross-over wiring required to build a realistic neural network would rise exponentially, that suggests exaggerated design sophistication and decreased fault sensitivity for specific network cells. The lower the semiconductor instruments, the greater the problems relating to cross-over insulation and trainable weights. Weights are usually used as managed storage appliances; however, weights are retrieved and configured by normal addressing logic. Although this strategy makes it easy to train, this may not necessarily the most ideal approach due to the introduction of hardware measurements.An alternative practical design that accurately represents the software neural network template will require an Association in nursing comprehensive methodology to hardware deployment whereby the specific cells are self-contained with their own assigned weights. Nonetheless, it cannot be a trivial problem, specifically while integrating giant-scale artificial neural networks, such as discussing reminiscence between each cell and communicating with network cells, thus continuing to need more control circuits that enhance the difficulty of design and execution. [3].

The latest expansion of the deep development method is that the gradable temporal networks (HTMs) shown in Fig One utilize deep frameworks in a highly gradable fashion. A high degree of functionality and dominance of quality and design could be a key characteristic of the cerebral mantle. HTM incorporates several of the prevalent pattern recognition concepts to construct a network solution to entity and series forecasting over time.

Memory networks solve these design issues that emerge with cross-over wiring and add a high-level of gradable extensibility that is invisible in traditional artificial neural networks. Recent performances suggest that gradable networks such as those "galvanized from plant tissue

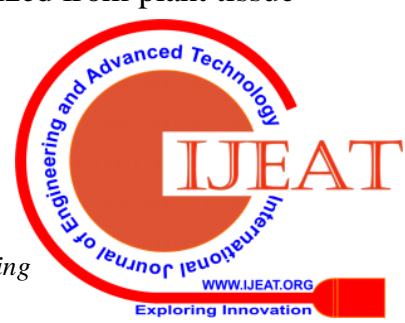


learning algorithms (e.g. HTM)" well-accomplish against shifts in "untrained natural variability". A quantity of small scale and popular software deployments has provided impressive results and observations into the field of gradable storage networks. [6]

Cognitive memory structures, on the other side, use a farreaching basic methodology to application and training. The expanded version of the memory framework described in is shown in Fig 2, where it is implemented to realistic facial recognition problems in real-time conditions. This implements a guided learning strategy utilizing different aspects of genetic testing to adjust cell weights. Cognitive memory network weights are comparable resistors for 2 and 3 terminal resistive memory units. The weights in a cognitive memory network are equivalent resistors of 2 or 3 terminal resistive memory devices. Since equivalent resistors of the memory elements are used for training the network, the actual use of memory elements as storage becomes implicative than formal. The network as a complete, through its different layers, memorizes the case, which ensures that it enables specific cell stability, allowing the network fault sensitive and resilient to shifts in natural variance. Therefore, scaling up these, a network to the degree that a typical human brain would like to go becomes just a trivial problem, which would be normally not feasible for traditional artificial neural-type network architectures.

These properties of the memory networks make it an effective alternative to comprehend and emulate intelligence in machines. Additionally, the hierarchical nature of the network can be reconnoitred to implement "image processing and data compression application", such as done with "autocorrelation neural networks".
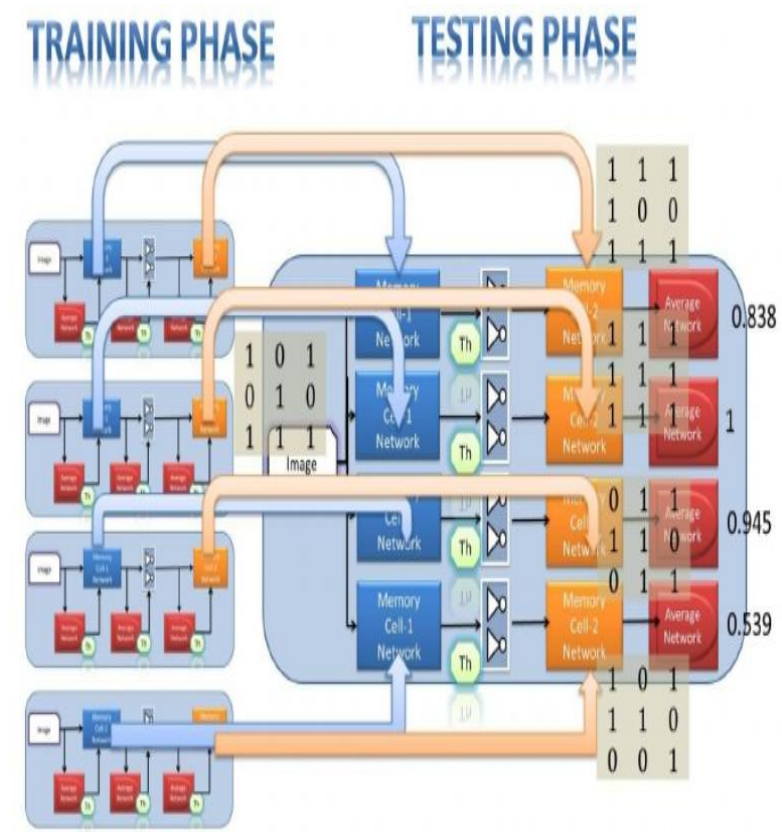

Fig .2.The block-level illustration of the memory network in a multiclass high-dimensional pattern recognition problem.

\section{FUTURE SCOPE}

The future potential effect of storage network architectures is that it will execute sensory recognition, vision, awareness, attention, and concentration functions. Although most of the network's data processing time relies on the most space access period used. As far as software integration is concerned, it includes remembrances that can do a large number of loops, less storage and long persistence of information that can be made achievable with "QsRAM".

The modularity of the network is sometimes used to build massive software systems, which will improve the versatility of the network to support a number of advanced features and the way artificial information is created. The versatility of the network to perform complicated tasks relies on the physical characteristic of the overall layout which essentially implies the specification of storage modules, surfaces, and the number of cell outputs that could result in a variation of network architectures.

The hardware memory infrastructure will have constructions that are higher or worse for the retardant it can accommodate. In particular, the selection of the network structure relies on the specific performance and complexity of the project. In the instances we've presented, what a simple cell type is going to do after we spread it across the network. The amount of outputs that can be wired to a cell is limited to a maximum of half a dozen owing to technical issues involved with an electricity converter.

In contrast, the nonlinear electrical converter that works inside the cell can induce irregularities in its performance that could migrate from one layer to the next. Nevertheless, modelling tests also demonstrate that these mistakes are insignificant and have no impact on the activity of the network. The network is resilient to the acceptance and vulnerability to resistance fluctuations, which ensures that minor differences of the qualified values do not have an impact on the functionalism of the network.

\section{CONCLUSION}

The data network architecture of "1397 inputs" and "13bit" output network on hardware needs " 1910 bits of data" and "274" inverters with an input ratio of $1 / 1345$ with triple tiers of cells [6].

So if we tend to extend the memory to one gigabit with twelve-bit output implies an input resolution of " $1 / 766958445$ " with ten layers of cells, and this network is going to be ready to solve the other advanced problems connected with the examples self-addressed.

The "106-gigabit memory network chip" would handle almost all of the functions in which an individual's brain would do, although recalling one or two gigabits would be sufficient to deal with some of the other brain's psychological features.

Current technologies offer storage for memory that can interact with biological recollections and is underutilized due to technological constraints. With many innovations in progress, large semiconductor recollections will be produced that can be accomplished in the coming years.

This can make it easier to build massive storage network chips that can equal the mental capacity of the human brain and could be the starting point for a truly functional, autonomous computer science. 


\section{REFERENCES}

1. H. T. Siegelmann and E. D. Sontag, "Analog computation via neural networks," Theor. Comput. Sci., 1994.

2. F. M. Dias, A. Antunes, and A. M. Mota, "Artificial neural networks: A review of commercial hardware," Eng. Appl. Artif. Intell., 2004.

3. S. DRAGHICI, "NEURAL NETWORKS IN ANALOG HARDWARE - DESIGN AND IMPLEMENTATION ISSUES,” Int. J. Neural Syst., 2003.

4. J. Hawkins, D. George, and J. Niemasik, "Sequence memory for prediction, inference and behaviour," Philos. Trans. R. Soc. B Biol. Sci., 2009.

5. D. George and J. Hawkins, "Towards a mathematical theory of cortical micro-circuits," PLoS Comput. Biol., 2009.

6. T. Klingberg, "Training and plasticity of working memory," Trends in Cognitive Sciences. 2010. 